\title{
Bidirectional modulation of hyperalgesia via the specific control of excitatory and inhibitory neuronal activity in the ACC
}

\author{
Sukjae Joshua Kang ${ }^{1}$, Chuljung Kwak ${ }^{2}$, Jaehyun Lee ${ }^{3}$, Su-Eon Sim¹', Jaehoon Shim', Taehyuk Choi ${ }^{3}$,
} Graham L. Collingridge $e^{4,5,6,7^{*}}$, Min Zhuo ${ }^{5,6^{*}}$ and Bong-Kiun Kaang ${ }^{2,5^{*}}$

\begin{abstract}
Neurons in the anterior cingulate cortex (ACC) are assumed to play important roles in the perception of nociceptive signals and the associated emotional responses. However, the neuronal types within the ACC that mediate these functions are poorly understood. In the present study, we used optogenetic techniques to selectively modulate excitatory pyramidal neurons and inhibitory interneurons in the ACC and to assess their ability to modulate peripheral mechanical hypersensitivity in freely moving mice. We found that selective activation of pyramidal neurons rapidly and acutely reduced nociceptive thresholds and that this effect was occluded in animals made hypersensitive using Freund's Complete Adjuvant (CFA). Conversely, inhibition of ACC pyramidal neurons rapidly and acutely reduced hypersensitivity induced by CFA treatment. A similar analgesic effect was induced by activation of parvalbumin (PV) expressing interneurons, whereas activation of somatostatin (SOM) expressing interneurons had no effect on pain thresholds. Our results provide direct evidence of the pivotal role of ACC excitatory neurons, and their regulation by PV expressing interneurons, in nociception.
\end{abstract}

\section{Background}

The role of the anterior cingulate cortex (ACC) in pain conditions has been consistently demonstrated for the past several decades [1, 2]. Human brain imaging studies report responses of the ACC, and related cortical areas, to acute nociceptive stimuli [3-6]. Moreover, surgical lesions of the cingulate cortex (cingulotomy) reduced the unpleasantness of pain [7-10]. Given the important role of ACC in pain, numerous animal studies have been performed in this region to reveal the pathways and molecular mechanism of nociception and chronic pain [11-13]. For example, lesioning of rat ACC, similar to human cingulotomy, reduced aversive behavior during the conditioned place aversion test [14], and escape/ avoidance test [15]. Numerous electrophysiological studies

\footnotetext{
* Correspondence: glcollingridge@gmail.com; min.zhuo@utoronto.ca; kaang@snu.ac.kr

${ }^{4}$ Centre for Synaptic Plasticity, School of Physiology and Pharmacology, University of Bristol, Bristol BS8 1TD, UK

${ }^{5}$ Center for Neuron and Disease, Frontier Institutes of Life Science and of Science and Technology, Xi'an Jiaotong University, Xi'an, China

${ }^{2}$ Department of Biological Sciences, College of Natural Sciences, Seoul

National University, Seoul 151-747, South Korea

Full list of author information is available at the end of the article
}

have also observed increased activation of the ACC in both acute and chronic pain situations. For example, an acute nociceptive stimulus in the peripheral region increased firing of ACC pyramidal neurons whereas a brush stroke had minimal effect [16-18]. Peripheral injury induced long-term potentiation (LTP) of excitatory synaptic responses in the ACC neurons $[19,20]$. Pharmacological inhibition or genetic deletion of LTP related molecules in the ACC reduced pain in chronic models [21-24]. Furthermore, using pharmacological manipulations and electrical stimulation within the ACC, it has been shown that ACC activation can elicit aversive behaviors [25, 26]. Yet, the spatiotemporal precision of ACC neuronal activity in pain remains enigmatic and the function of different ACC neuronal subtypes is controversial.

Optogenetic tools are increasingly being used to identify the neural circuits underlying various types of behaviors or to reveal the functions of specific subtypes of cells $[27,28]$. Several cre line mice are available for the selective manipulation of specific cell types [29, 30]. However, these methods have only just started to be applied to the understanding of pain pathways. Studies to date have been 
performed in peripheral regions [31], brain stem [32], medial prefrontal cortex (mPFC) [33-35] and ACC $[36,37]$. The two optogenetic studies in the ACC used Thy1-ChR2 mice which expresses ChR2 indiscriminately within excitatory and inhibitory neurons. One group reported that activation of ACC showed anxiodepressivelike behaviors but no change in pain [36]. However, the other group observed an alleviation of acute inflammatory pain [37] which they attributed to the activation of inhibitory interneurons. The reason for these discrepant results is unknown.

In the present study, we used optogenetics and creexpressing mouse lines to, for the first time, specifically manipulate the activity of excitatory neurons and interneurons within the ACC to establish whether specific neuronal subtypes within this brain region can acutely modulate nociceptive responses. We injected channelrhodopsin-2 (ChR2) or halorhodopsin (eNpHR3.0) carrying adenoassociated virus (AAV) in CaMKII-cre mice to activate or inhibit, respectively, the CAMKII-expressing ACC excitatory neurons. We also injected ChR2 into PV-cre or SOM-cre mice to activate specific PV positive or SOM positive interneurons. We found that activation of ACC pyramidal neurons decreased the basal mechanical threshold but did not further decrease the level in CFA-treated mice. In contrast, inhibition of these neurons reversed the effects of CFA treatment without affecting the basal mechanical threshold. Moreover activation of PV-type interneurons, but not SOM-type interneurons, also alleviated the CFA-induced pain hypersensitivity. These results show that excitatory neurons within the ACC are both necessary and sufficient for nociceptive processing and that they are under tight regulation by PV-expressing interneurons. These results suggest that ACC excitatory neurons are one of the critical mediators of nociception.

\section{Methods \\ Animals}

Adult (8-12 week old) male CaMKII-, PV- or SOM- cre mice (Jackson Laboratory) were used. All animals were housed under a $12 \mathrm{~h}$ light/dark cycle with food and water provided ad libitum. All works were conducted according to the policy and regulation for the care and use of laboratory animals approved by Institutional Animal Care and Use Committee at Seoul National University.

\section{Stereotaxic virus injection, optic cannula implantation} AAV carrying DIO-ChR2-EYFP, DIO-eNPHR3.0-EYFP or DIO-EYFP constructs was injected into 6 week-old CaMKII-cre hetero male mice. PV-cre hetero and SOMcre hetero or wild-type littermates (control) were also injected with AAV-DIO-ChR2-EYFP. Mice were anesthetized with an intraperitoneal injection of ketaminexylazine $(0.1 \mathrm{mg}$ per gram body weight ketamine, $0.01 \mathrm{mg}$ per gram body weight xylazine) and the head was fixed in a stereotaxic apparatus (Kopf Instruments, Tujunga, CA, USA). A small craniotomy was performed and, five holes were drilled. Two holes were drilled bilaterally between the hippocampus and cerebellum for screw implantation. The other three holes were drilled for virus injection and optic cannula implantation in the ACC. The virus was delivered using a $10 \mu \mathrm{l}$ syringe (Hamilton) and a 30 gauge metal needle. The injection volume and flow rate $(0.5 \mu \mathrm{l}$ at $0.1 \mu \mathrm{l} / \mathrm{min}$ ) were controlled by an injection pump (WPI). Virus was injected into both side of the ACC (anteroposterior $[\mathrm{AP}]+1.0 \mathrm{~mm}$ from bregma, mediolateral $[\mathrm{ML}] \pm$ $0.35 \mathrm{~mm}$, dorsoventral [DV] $-2.2 \mathrm{~mm})$. After injection, the needle was left for additional $7 \mathrm{~min}$ and then was slowly removed. The optic cannula (MFC_200/230-0.39 2mm_ZF1.25_FLT, Doric Lenses., Quebec, Canada) was implanted between the virus injection site $(\mathrm{AP}+1.0 \mathrm{~mm}$ from bregma, $\mathrm{ML}+0 \mathrm{~mm}, \mathrm{DV}-1.25 \mathrm{~mm}$ ). The optic cannula was then fixed with dental cement.

\section{Behavior \\ Electrical von Frey system for checking the mechanical threshold}

A pre-test was done 3 weeks after the surgery and CFA (1:1 mixture with saline) or saline $10 \mu \mathrm{l}$ was injected in the hind paw after the test. 3 days later the post-test was performed. In each test, there were three light 'off' and two light 'on' sessions (off1-on1-off2-on2-off3, $5 \mathrm{~min}$ intersession interval). Paw withdrawal threshold was measured with the electronic von Frey system. The averages of three values were calculated as the threshold of that session. $593 \mathrm{~nm}$ light was given continuously in an intensity of $7-9 \mathrm{~mW} / \mathrm{cm}^{2} .473 \mathrm{~nm}$ light was given at $10 \mathrm{~Hz}$ (40 ms pulse) throughout the 'on' period for the CaMKII-cre mice. PV-cre and SOM-cre mice were given $473 \mathrm{~nm}$ light in $300 \mathrm{~ms}$ pulses with $2 \mathrm{~s}$ inter-pulse intervals. An intensity of $20-25 \mathrm{~mW} / \mathrm{cm}^{2}$ at fiber tip was used in all ChR2 experiments.

\section{Open field test}

Mice were exposed for 15 min to the open field apparatus under dim light. The open field was a square opaque white box $(40 \times 40 \times 40 \mathrm{~cm})$ in which mice were monitored with a tracking program (EthoVision 3.1, Noldus, Netherlands). Light stimulation was given throughout the experiment.

\section{Electrophysiology}

Patch clamp recording

For whole-cell patch clamp recording, $300 \mu \mathrm{m}$ thick coronal slices of ACC were prepared with a Leica VT-1000S slicer and incubated in artificial cerebrospinal fluid (aCSF) at $25 \sim 26^{\circ} \mathrm{C}$ for $1 \mathrm{~h}$. The aCSF comprised $124 \mathrm{mM} \mathrm{NaCl}$, $2.5 \mathrm{mM} \mathrm{KCl}, 1 \mathrm{mM} \mathrm{NaH} \mathrm{PO}_{4}, 25 \mathrm{mM} \mathrm{NaHCO}, 10 \mathrm{mM}$ 
Glucose, $2 \mathrm{mM} \mathrm{CaCl}_{2}, 2 \mathrm{mM} \mathrm{MgSO}$, was used for the incubation and bath solution. The bath solution was oxygenated with $95 \% \mathrm{CO}_{2}, 5 \% \mathrm{O}_{2}$ mixed gas and perfused $2 \mathrm{ml} / \mathrm{min}$ at $25 \sim 26{ }^{\circ} \mathrm{C}$ (TC-324B, Warner). The slices were transferred to the recording chamber of a BX51WI microscope (Olympus) and visualized with a ProgRes MFcool CCD camera.

For current-clamp recording, the borosilicate glass recording pipettes were filled with internal solution containing $145 \mathrm{mM}$ K-gluconate, $5 \mathrm{mM} \mathrm{NaCl}, 0.2 \mathrm{mM}$ EGTA, $10 \mathrm{mM}$ HEPES, $2 \mathrm{mM}$ MgATP, $0.1 \mathrm{mM} \mathrm{Na}_{3} \mathrm{GTP}, 1 \mathrm{mM}$ $\mathrm{MgCl}_{2}$ (pH 7.2 with $\mathrm{KOH}, 280 \sim 290 \mathrm{mOsm}$ ). For current injection, pyramidal neurons in layer $2 / 3$ were current clamped and $20 \mathrm{pA}-140 \mathrm{pA}$ depolarizing current was injected for $10 \mathrm{~s}$ while $473 \mathrm{~nm}$ light was delivered to the $\mathrm{ACC}$ area using a $300 \mathrm{~ms}$ pulse width and $2 \mathrm{~s}$ inter-pulse interval. For pulse train experiments, pyramidal neurons in layer $2 / 3$ were current clamped and $2 \mathrm{~Hz}$ depolarizing current pulses (80 pA pulse with 200 ms width) were injected for 90 s. $473 \mathrm{~nm}$ light was delivered to the ACC area using $300 \mathrm{~ms}$ pulses with a $2 \mathrm{~s}$ inter-pulse interval for $30 \mathrm{~s}$ in the middle of the trains.

\section{In-vivo recording}

After 3 weeks of recovery, the mice was anesthetized with urethane (Sigma, $0.13 \mathrm{~g} / 100 \mathrm{~g}$ ) injection and the head was fixed in a stereotaxic apparatus (Kopf Instruments, Tujunga, CA, USA). A small craniotomy was performed and two holes were drilled above the ACC on each side of the brain (AP $+1.0 \mathrm{~mm}$ from bregma, $\mathrm{ML} \pm$ $0.0 \mathrm{~mm}$ ) and a third was drilled in the cerebellum for reference. A customized optrode, composed of a tungsten electrode (A-M Systems, USA) and optic cannula (MFC_200/230-0.39_50mm_ZF1.25_FLT, Doric lenses, USA), was inserted into the ACC for multiunit recording. Neural activity was amplified 1000 fold and digitized (sampled at $32 \mathrm{kHz}$, filtered at $600-6000 \mathrm{~Hz}$ ) using a Digital Lynx data acquisition system (Neuralynx).

\section{Data analysis}

All data are presented as mean \pm SEM. Statistical comparisons were made using the $t$-test, one-way and two-way ANOVA by SigmaPlot 11.0. The post hoc Bonferroni test was used for further comparison. If the data did not pass the equal variance test, one way ANOVA was done in ranks and Dunn's method was used for post-hoc test. In all cases, statistical significance was indicated by " $p<0.05$.

\section{Results}

Discrete cellular population in the ACC and manipulation of the excitatory neurons

In the present study, we used three cre mouse lines (CaMKII-, PV-, SOM-cre) for optogenetic manipulation in the ACC. We performed immunostaining in the ACC to observe the distribution of these three different molecular targets. AAV-DIO-EYFP was injected bilaterally into the ACC of CaMKII-cre mice and PV and SOM were stained with antibodies (Fig. 1a, Additional file 1: Figure S1). All three markers were expressed throughout the ACC but within discrete cellular populations.

We next tested the effectiveness of the rhodopsins in pyramidal neurons within the ACC. AAV-DIO-eNpHR3.0, AAV-DIO-ChR2 or AAV-DIO-EYFP were injected into the ACC of CaMKII-cre mice and multiunit in vivo recording using an optrode was performed 3 weeks later. Activation of ChR2 with blue (473 nm) light stimulated the ACC neurons whereas activation of eNpHR3.0 with yellow (593 nm) light inhibited their activity (Fig. 1b).

\section{Activation of excitatory neurons in the ACC decreases the mechanical threshold}

Throughout our study, we injected AAV-DIO-ChR2, AAV-DIO-eNpHR3.0 or AAV-DIO-EYFP in the ACC bilaterally and the optic cannula was implanted in the middle of the viral injection sites (Fig. 2a). We first tested whether specific activation of ACC excitatory neurons is itself sufficient to modulate the nociceptive responses. To address this question, AAV-DIO-ChR2 was injected into the ACC of CaMKII-cre mice (CaMKII-ChR2) and $473 \mathrm{~nm}$ light was pulsed at $10 \mathrm{~Hz}$ throughout the 'ON' period for ACC activation. This frequency was selected according to the previous in vivo whole-cell patch recording data in the ACC during noxious stimulation [16]. Interestingly, the mechanical threshold of the majority $(10 / 18)$ of the mice decreased when the light was given and fully recovered back to the baseline value when the light was turned off (Fig. 2b, d). This effect was replicated when a second period of light excitation was employed (Fig. 2b, d). In contrast, the AAV-DIO-EYFP injected control group was invariably unaffected by light (Fig. 2b) [EYFP (off: $4.99 \pm 0.22 \mathrm{~g}$, on: $4.81 \pm 0.22 \mathrm{~g} ; n=10$ ) and ChR2 (off: $4.90 \pm 0.13$ g, on: $4.12 \pm 0.22$ g; $n=18$; Fig. 2c)]. The 'off' and 'on' sessions were averaged for statistical analysis and there was a statistically significant interaction between light ('off' and 'on') and virus ('ChR2' and 'EYFP') infused ( $p=0.011$, two-way repeated measures ANOVA). There was no difference in the patterns of ChR2 expression between the "decrease" and "non-decrease" groups (Fig. 2e).

\section{Activation of ACC excitatory neurons has no effect in chronic pain conditions}

To examine the role of ACC excitatory neurons in chronic pain condition, we used CFA treatment and performed behavioral experiments as shown schematically in Fig. 3a. In contrast to the effects of activating ACC excitatory neurons of CaMKII-ChR2 mice in a non- painful situation (Before CFA; off: $5.00 \pm 0.23 \mathrm{~g}$, on: $4.13 \pm 0.40 \mathrm{~g} ; n=8$; 


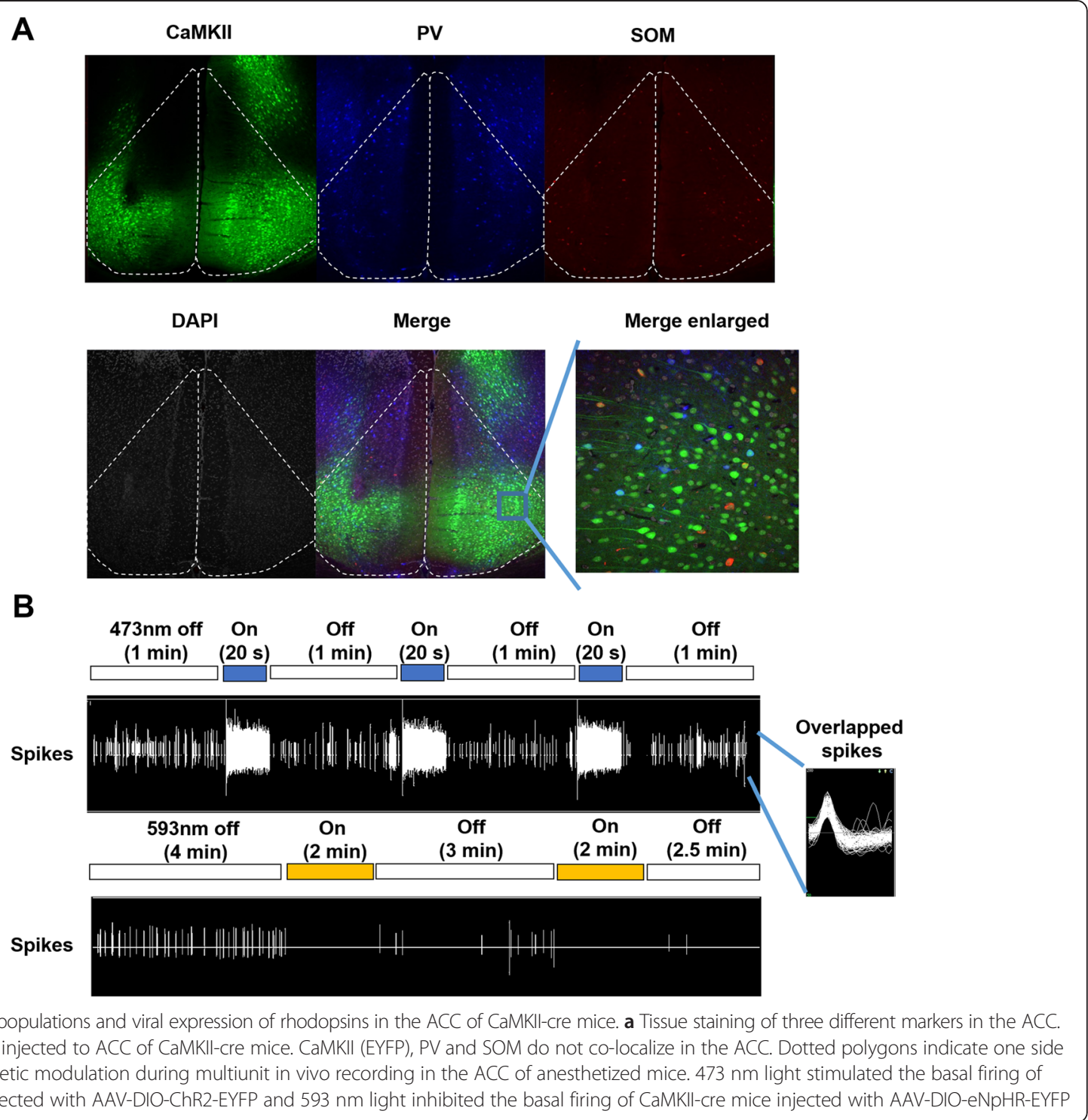

Fig. 3b black), there was no modification of the mechanical threshold in the chronic pain state (After CFA; off: $3.25 \pm$ $0.25 \mathrm{~g}$, on: $3.29 \pm 0.28 \mathrm{~g} ; n=8$; Fig. $3 \mathrm{~b}$ red). This suggests an occlusion of the effects of optogenetic activation of excitatory ACC neurons and the effects of inflammatory paininduced signals. In the saline injected control group, there was a similar light-induced reduction in the pain threshold both before (off: $4.83 \pm 0.15 \mathrm{~g}$, on: $4.12 \pm 0.25 \mathrm{~g} ; n=10$; Fig. 3c green) and after saline (off: $4.54 \pm 0.11 \mathrm{~g}$, on: $4.08 \pm$ 0.23 g; $n=10$; Fig. $3 c$ blue) injection. There was no light effect in control groups (Additional file 2: Figure S2).

To test the effects of ChR2 activation on locomotion and anxiety we performed an open field test (OFT). We found that there were no difference between the CaMKIIEYFP and CaMKII-ChR2 groups in the OFT when light was given throughout the experiment. (Additional file 4: Figure S4A). These results identify excitatory ACC neurons as effectors of pain responses and show that their activation alone is sufficient for a significant alteration in the mechanical pain threshold.

\section{Inhibition of excitatory neurons in the ACC reverses the} effects of inflammatory pain

We next inhibited ACC excitatory neurons, expressing eNpHR3.0 (CaMKII-eNpHR), with yellow light and determined the mechanical threshold before and 3 days after CFA treatment. Interestingly, optogenetic illumination had no effect on the mechanical threshold before CFA injection (off: $4.94 \pm 0.22$ g, on: $4.90 \pm 0.36$ g; $n=9$ ) but after CFA treatment it reversed the mechanical pain threshold to near control values $(3.05 \pm 0.19 \mathrm{~g}$, on: $4.16 \pm 0.24 \mathrm{~g} ; n=9$; Fig. $4 \mathrm{a}$ ). The effect of optogenetic illumination was fully reversible and repeatable. Thus, optogenetic inhibition of excitatory neurons in the 


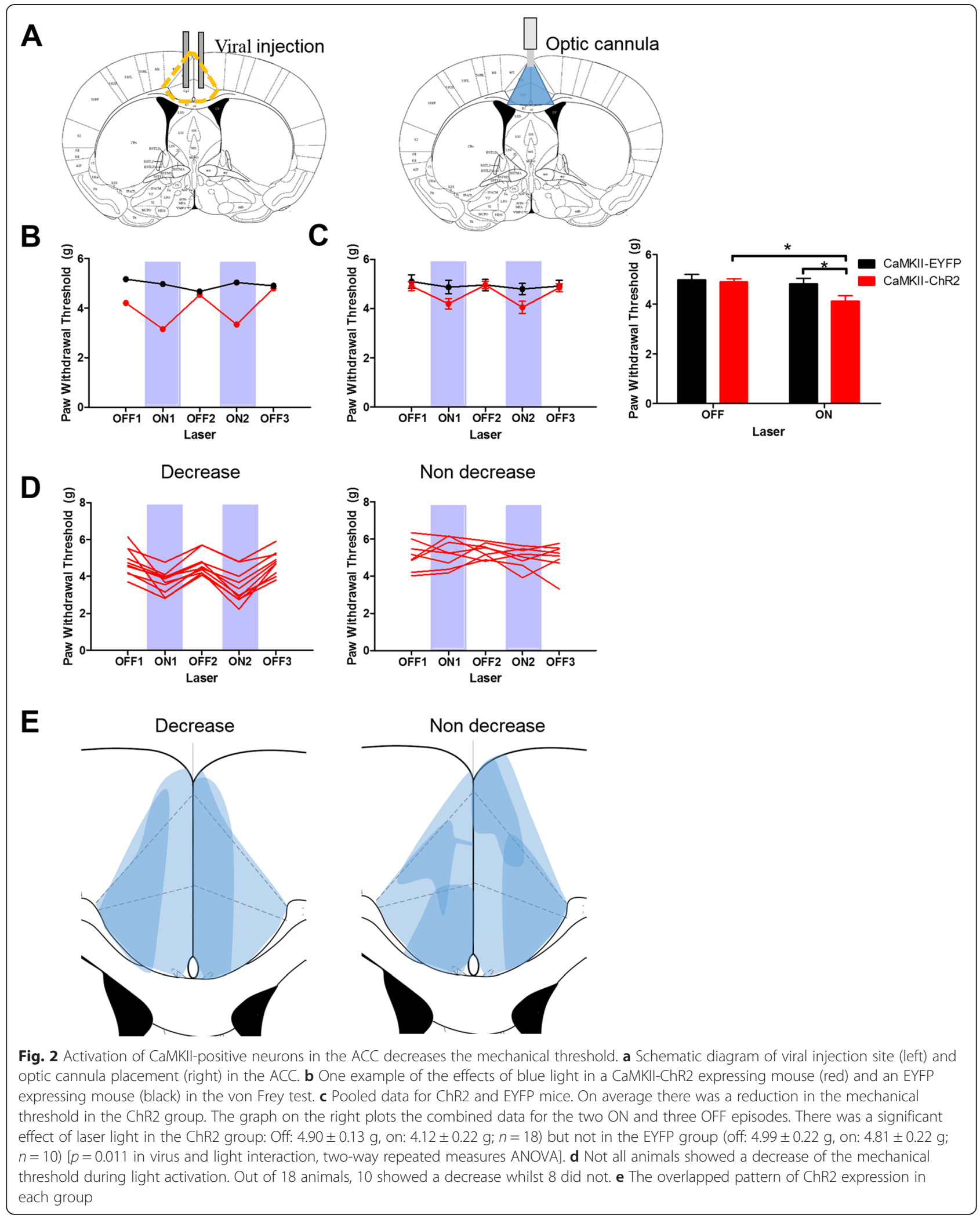




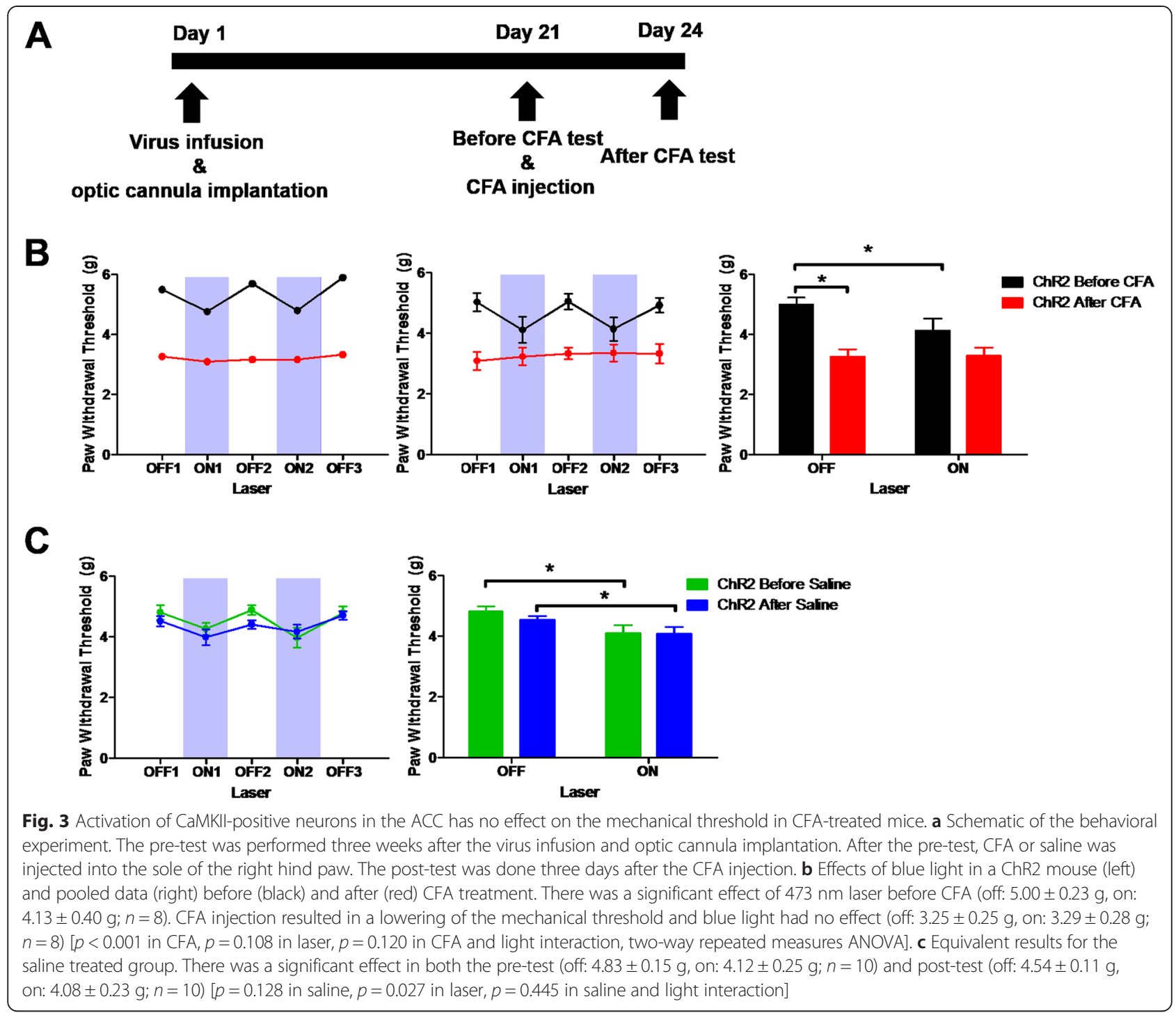

ACC substantially reversed the increase of mechanical sensitivity in the chronic inflammation pain model $(p=$ 0.011 in drug and light interaction, two-way ANOVA repeated measures). In contrast, there was no effect of light in the saline injection group and other control groups (Fig. 4b, Additional file 3: Figure S3 ). Comparison of the light 'ON' phases for all four groups showed statistically significant differences between the groups $(p<0.001$, one-way ANOVA), such that the EYFP-CFA group was significantly different from the other groups (Fig. 4c). To test whether the inhibition affects locomotion and anxiety we performed the OFT but could not see any difference between CaMKII-EYFP and CaMKII-eNpHR groups (Additional file 4: Figure S4B).

To test for the specificity of excitatory neurons within the ACC for the modulation of the pain threshold, we examined the effects of light on CaMKII-eNpHR neurons within the restrospenial cortex (RSG), a region adjacent to the ACC. In contrast to the ACC, yellow light had no effect on pain thresholds (Additional file 5: Figure S5). These result shows that transient inhibition of excitatory neurons in the ACC can significantly reverse the enhanced sensitivity to a mechanical stimulus caused by inflammation. This suggests, therefore, that excitatory neurons in the ACC may be effectors of the sensation of pain.

Optogenetic activation of inhibitory neurons in the ACC reverses the effects of inflammatory pain

The ACC comprises both excitatory neurons and a diverse array of inhibitory neurons that may play distinct roles in the modulation of pain thresholds. A major class of GABAergic inhibitory neurons expresses parvalbumin (PV). We therefore tested whether activation of the ACC PV-positive interneurons could modulate the mechanical threshold during chronic inflammation pain. $473 \mathrm{~nm}$ light 


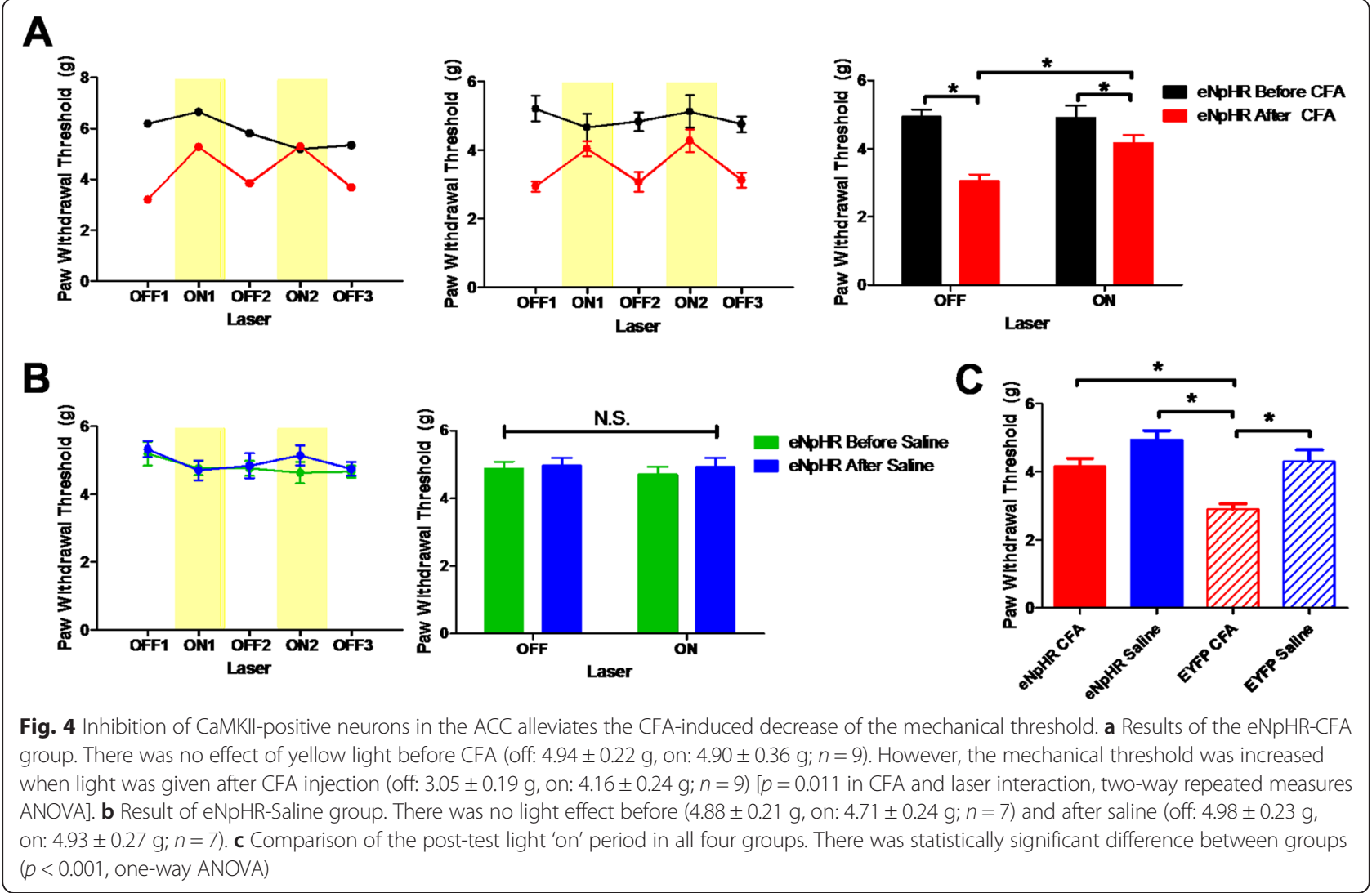

was given at $0.5 \mathrm{~Hz}$ throughout the $\mathrm{ON}$ period, using a protocol previously applied to the amygdala [30]. We found that activation of PV-ChR2 expressing neurons could substantially alleviate CFA-induced pain. In the PVChR2 group there was no significant effect of the $473 \mathrm{~nm}$ light before CFA (off: $5.30 \pm 0.15$ g, on: $5.04 \pm 0.07$ g; $n=6)$. However, there was significant increase in the mechanical threshold after CFA injection (off: $3.44 \pm$ $0.11 \mathrm{~g}$, on: $4.55 \pm 0.21 \mathrm{~g} ; n=6$; Fig. $5 \mathrm{a} ; p=0.002$ in CFA and light interaction, two-way repeated measures ANOVA). Light had no effect in other control groups (Additional file 6: Figure S6). Since the behavioral outcome is similar to that of the CaMKII-eNpHR mice, we wondered whether PV optogenetic activation will inhibit excitatory neurons. Current injection and pulse train tests were performed using whole-cell patch-clamp recording and light was given at $0.5 \mathrm{~Hz}$ to mimic the behavioral experiments with the PV-ChR2 mice. In the current injection experiment, (Fig. 5b, right), excitatory neurons showed a greater decrease of firing in the light group compared to the control group (Fig. 5b, left). Moreover, in the pulse train test, the firing of excitatory neurons were reduced during the light presentation and recovered when the illumination was stopped (Fig. 5c). These results demonstrate that optogenetic excitation of $\mathrm{PV}+$ interneurons inhibits the activity of ACC pyramidal neurons.
In contrast to the effects of activation of PV+ interneurons, there was no effect of the illumination of SOMChR2 expressing neurons on the mechanical threshold either before (off: $5.11 \pm 0.15$ g, on: $4.95 \pm 0.11 \mathrm{~g} ; n=12$ ) or after CFA treatment (off: $3.32 \pm 0.16 \mathrm{~g}$, on: $3.30 \pm$ 0.13 g; $n=12$; Fig. 5 d, Additional file 7: Figure S7). The whole-cell patch-clamp experiments also showed minimal effect of optogenetic excitation of SOM+ interneurons on the activity of ACC pyramidal neurons (Fig. 5e, f). These results show that the selective activation of $\mathrm{PV}+$ interneurons, but not SOM+ interneurons, in the ACC is able to reverse the effects of inflammation on the mechanical pain threshold, without affecting the mechanical pain threshold under basal conditions.

\section{Discussion}

In the present study, we have demonstrated that neurons within the ACC are acutely involved in the nociceptive responses of painful stimuli. We found that the specific activation of excitatory neurons in the ACC results in a lowering of the mechanical pain threshold, which is consistent with the view that the ACC is important in nociceptive responses $[1,2,12,16]$. Interestingly, the effect occurred rapidly upon optogenetic excitation, was rapidly reversible upon termination of illumination and was reproducible upon a second trial. This strongly suggests 


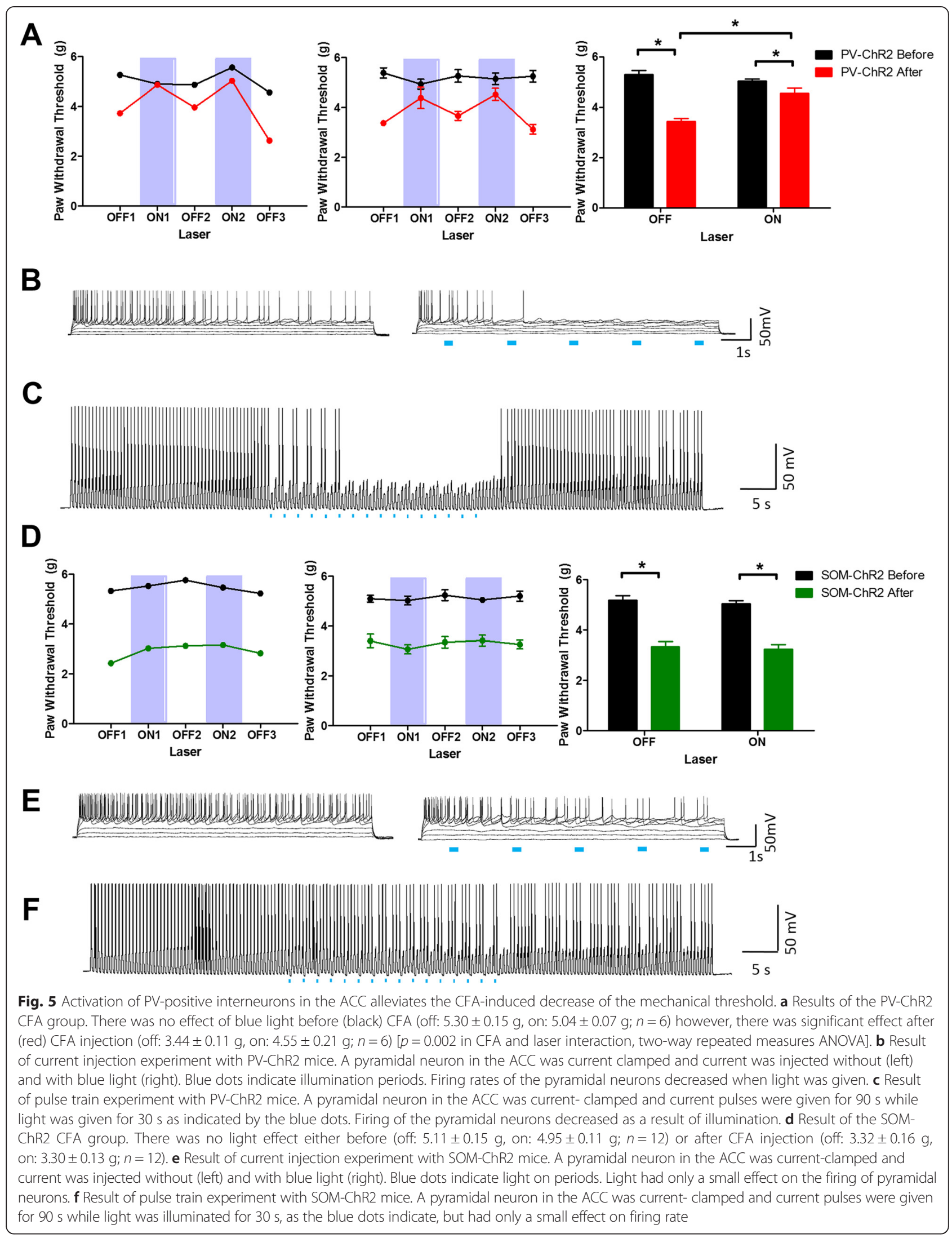


that these ACC neurons are effectors of the nociceptive responses as opposed to being a secondary reporter of the stimulus. This may be due to activating the descending facilitation projection [38]. Of course, our findings do not exclude the possibility that there are other primary mediators of the nociceptive responses. However, the observation that simply exciting a proportion of excitatory neurons in the ACC was able to significantly modify the mechanical pain threshold demonstrates the key role played by these neurons.

The observation that optogenetic excitation of excitatory ACC neurons was unable to further lower the mechanical pain threshold in mice treated with CFA suggests an occlusion between the two effects. In other words, an increase in activity in these ACC neurons may be mediating the nociceptive responses of inflammatory pain. If this is the case, the prediction would be that optogenetic inhibition of this neuronal may reverse the effects of inflammation on the mechanical pain threshold. The finding that optogenetic inhibition of excitatory ACC neurons had a substantial effect on the mechanical threshold after CFA treatment strongly suggests that these neurons do indeed convey this nociceptive signal. Interestingly, optogenetic inhibition of these neurons had no effect under normal (non-inflammatory) conditions. Thus, an increase in activity of this neuronal population signals the hyperalgesic state. When considered together these results imply that excitatory neurons in the ACC are both necessary and sufficient to mediate sensitization to mechanical stimuli.

Excitatory neurons in the cortex are under strong regulation by GABAergic interneurons. We found that optogenetic stimulation of one class, PV-containing interneurons, was sufficient to have a substantial effect on the pain threshold. This effect was again specific for the inflamed state. Indeed, in all respects, stimulation of this single interneuron class mimicked the effects of inhibition of the excitatory neurons within this brain structure. This observation is consistent with the role of PV+ interneurons as mediators of powerful feed-forward inhibition of pyramidal neurons [39]. It shows that excitatory ACC neurons are under powerful influence of this interneuronal subclass during painful stimuli and that their specific activation is sufficient to alleviate mechanical hypersensitivity, presumably by their inhibition of pyramidal neurons in the ACC. Recently, an opposite conclusion has been reached with respect to the role of the $\mathrm{mPFC}$, especially the prelimbic cortex (PL), in the processing of painful information. Activation of PL excitatory neurons reduced pain [34], and activation of PV interneurons induced more pain [35]. Moreover, activation of PL projection to nucleus accumbens relieved pain [33]. These results highlight how anatomically distinct cortical regions have discrete roles in pain processing.
Recently two other studies have used optogenetic approaches to address specifically the functional roles of the ACC but they drew different conclusions. In one study it was reported that optogenetic stimulation of Thy1-ChR2 mice did not affect the mechanical threshold [36] whereas in the other study it was reported that activation of Thy1ChR2 mice resulted in analgesia [37]. Why the two studies reached different conclusions is unclear, though it may be due to the use of different Thy1-ChR2 mice lines. There are several possibilities why our conclusions differs from the study of Barthas et al. [36]. Firstly, in the Thy1 mouse there is expression of ChR2 in multiple neuronal types. Indeed, we found that specific activation of ChR2 in pyramidal neurons and PV+ interneurons had diametricallyopposed effects. Secondly, in this previous study, optogenetic stimulation and the mechanical threshold test were not performed at the same time. Therefore, this raises the possibility that chronic activation of ACC excitatory neurons does not affect the mechanical threshold but rather this requires their acute activation. Our study does, however, agree with the report of $\mathrm{Gu}$ et al. [37], which attributed the analgesia to the activation of inhibitory neurons, and extends these findings by identifying PV+ neurons as a critical inhibitory neuronal class.

In the study by Barthas et al. [36] it was reported that optogenetic stimulation resulted in a long lasting alterations in anxiety and depressive-like behaviors [36]. In the present study we did not observe any effects of optogenetic manipulation on anxiety-like behavior, as assessed using the open field test. This difference could be explained by the need for repeated stimulation to elicit mood-related behaviors and/or because in the Thy1-ChR2 mice mixed cell types are affected.

\section{Conclusions}

In conclusion, we have identified two specific neuronal types within the ACC that are involved in the nociceptive responses to inflammatory pain. These results raise the possibility that specifically targeting these neuronal populations may lead to effective treatments of painful states.

\section{Additional files}

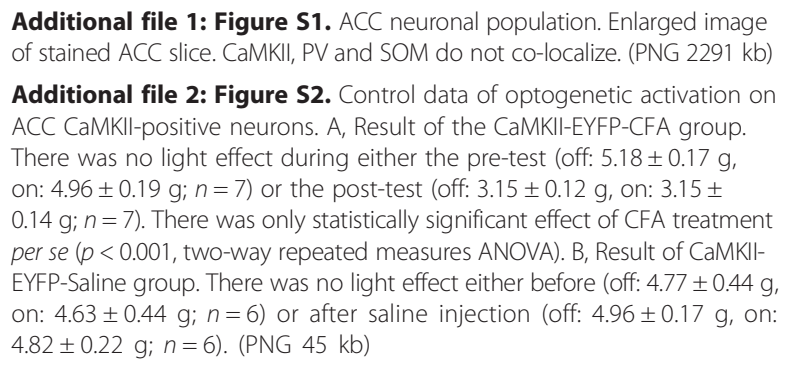

Additional file 1: Figure S1. ACC neuronal population. Enlarged image of stained ACC slice. CaMKII, PV and SOM do not co-localize. (PNG 2291 kb)

Additional file 2: Figure S2. Control data of optogenetic activation on ACC CaMKII-positive neurons. A, Result of the CaMKII-EYFP-CFA group. There was no light effect during either the pre-test (off: $5.18 \pm 0.17 \mathrm{~g}$, on: $4.96 \pm 0.19 \mathrm{~g} ; n=7$ ) or the post-test (off: $3.15 \pm 0.12 \mathrm{~g}$, on: $3.15 \pm$ $0.14 \mathrm{~g} ; n=7)$. There was only statistically significant effect of CFA treatment per se $(p<0.001$, two-way repeated measures ANOVA). B, Result of CaMKIIEYFP-Saline group. There was no light effect either before (off: $4.77 \pm 0.44 \mathrm{~g}$, on: $4.63 \pm 0.44 \mathrm{~g} ; n=6$ ) or after saline injection (off: $4.96 \pm 0.17 \mathrm{~g}$, on: $4.82 \pm 0.22 \mathrm{~g} ; n=6)$. (PNG $45 \mathrm{~kb}$ )

Additional file 3: Figure S3. Control data of optogenetic inhibition on ACC CaMKII-positive neurons. A, Result of the CaMKII-EYFP-CFA group. 
There was no light effect before (off: $4.77 \pm 0.20 \mathrm{~g}$, on: $4.97 \pm 0.15 \mathrm{~g}$; $n=6$ ) or after CFA (off: $2.81 \pm 0.22 \mathrm{~g}$, on: $2.90 \pm 0.16 \mathrm{~g} ; n=6$ ). There was only a statistically significant effect of CFA treatment per se $(p<0.001$, two-way repeated measures ANOVA). B, Result of CaMKII-EYFP-Saline group. There was no light effect either before (off: $4.44 \pm 0.30 \mathrm{~g}$, on: $4.17 \pm 0.28 \mathrm{~g} ; n=5$ ) or after CFA (off: $4.27 \pm 0.22 \mathrm{~g}$, on: $4.31 \pm 0.24 \mathrm{~g}$; $n=5)$. (PNG $42 \mathrm{~kb}$ )

Additional file 4: Figure S4. Control experiments for locomotion and anxiety. A, Results of the CaMKII-EYFP and CaMKII-ChR2 groups in the open field test (OFT). There was no difference between the two groups in either locomotion (EYFP: $5824 \pm 457.6 \mathrm{~cm}, n=8 ;$ ChR2: $6207 \pm 244.8 \mathrm{~cm}$, $n=17 ; p=0.4273$, Unpaired $t$ test) or time spent in the center (EYFP: $9.17 \pm 1.85 \%, n=8$; ChR2: $11.27 \pm 1.68 \%, n=17 ; p=0.4559$, Unpaired $t$ test). B, Results of the CaMKII-EYFP and CaMKII-eNpHR groups in the OFT. There was no difference between the two groups in either locomotion (EYFP: $6616 \pm 278.4 \mathrm{~cm}, n=7$; eNpHR: $6043 \pm 212.9 \mathrm{~cm}, n=12$; $p=0.1209$, Unpaired $t$ test) or time spent in the center (EYFP: $10.96 \pm 2.34 \%$, $n=7$; eNpHR: $10.15 \pm 1.35 \%, n=12 ; p=0.7488$, Unpaired $t$ test). (PNG $28 \mathrm{~kb}$ )

Additional file 5: Figure S5. Optogenetic inhibition of CaMKII-positive neurons in the retrospenial cortex (RSG). There was no light effect either before (off: $5.07 \pm 0.07 \mathrm{~g}$, on: $5.29 \pm 0.18 \mathrm{~g} ; n=8$ ) or after CFA injection (off: $3.28 \pm 0.13 \mathrm{~g}$, on: $3.38 \pm 0.19 \mathrm{~g} ; n=8$ ). (PNG $21 \mathrm{~kb}$ )

Additional file 6: Figure $\mathbf{S 6}$. Control data of optogenetic activation of ACC PV-positive interneurons. A, Result of the PV-ChR2 saline group. There was no light effect either before (off: $5.09 \pm 0.22 \mathrm{~g}$, on: $4.84 \pm 0.09 \mathrm{~g}$; $n=6$ ) or after saline injection (off: $5.18 \pm 0.18 \mathrm{~g}$, on: $4.79 \pm 0.23 \mathrm{~g} ; n=6$ ). B, Result of the WT-ChR2 CFA group. There was no light effect before (off: $5.18 \pm 0.13 \mathrm{~g}$, on: $5.12 \pm 0.12 \mathrm{~g} ; n=8$ ) or after CFA (off: $3.33 \pm 0.12 \mathrm{~g}$, on: $3.58 \pm 0.19 \mathrm{~g} ; n=8)$. There was only statistically significant effect of CFA treatment per se $(p<0.001$, two-way repeated measures ANOVA). C, Result of the WT-ChR2 saline group. There was no light effect either before (off: $4.7 \pm 0.15 \mathrm{~g}$, on: $4.93 \pm 0.28 \mathrm{~g} ; n=5$ ) or after saline (off: $4.94 \pm 0.15 \mathrm{~g}$, on: $4.67 \pm 0.27 \mathrm{~g} ; n=5$ ). (PNG $70 \mathrm{~kb}$ )

Additional file 7: Figure S7. Control data of optogenetic activation of ACC SOM-positive interneurons. Result of WT-ChR2 CFA group. There was no light effect either before (off: $5.15 \pm 0.20 \mathrm{~g}$, on: $5.32 \pm 0.20 \mathrm{~g} ; n=6$ ) or after CFA injection (off: $3.10 \pm 0.09 \mathrm{~g}$, on: $3.24 \pm 0.07 \mathrm{~g} ; n=6$ ). (PNG $23 \mathrm{~kb}$ )

\section{Competing interests}

The authors declare that they have no competing interests.

\section{Authors' contributions}

SK carried out the in vivo electrophysiology, surgery, behavioral experiments and drafted the manuscript. SS, CK and TC helped surgical procedures. TC carried out the immunostaining. $J L$ performed behavioral experiments. SS and JS carried out the whole cell patch clamp experiments. GLC, MZ and BKK helped to draft the manuscript. SK and BKK participated in the design of the study. All authors read and approved the final manuscript.

\section{Acknowledgements}

This work was supported by the Ministry of Education, Science and Technology in Korea through National Research Foundation (R32-10142). S.J.K. was supported by BK21 fellowship. B.-K.K. is a supported by the National Honor Scientist Program of Korea. MZ supported by Canada Research Chair, and Canadian Institute for Health Research operating grants (MOP-124807) and NSERC Discovery Grant (RGPIN 402555). GLC was supported by the MRC (UK).

\section{Author details}

${ }^{1}$ Department of Brain and Cognitive Sciences, College of Natural Sciences, Seoul National University, Seoul 151-746, South Korea. ${ }^{2}$ Department of Biological Sciences, College of Natural Sciences, Seoul National University, Seoul 151-747, South Korea. ${ }^{3}$ Interdisciplinary Program in Neuroscience, College of Natural Sciences, Seoul National University, Seoul 151-747, South Korea. ${ }^{4}$ Centre for Synaptic Plasticity, School of Physiology and Pharmacology, University of Bristol, Bristol BS8 1TD, UK. ${ }^{5}$ Center for Neuron and Disease, Frontier Institutes of Life Science and of Science and Technology, Xi'an Jiaotong University, Xi'an, China. ${ }^{6}$ Department of Physiology, Faculty of Medicine, University of Toronto, 1 King's College Circle,
Toronto, ON M5S 1A8, Canada. 'Lunenfeld-Tanenbaum Research Institute, Mount Sinai Hospital, Ontario M5G 1X5, Toronto, ON M5S 1A8, Canada.

Received: 17 November 2015 Accepted: 19 November 2015 Published online: 02 December 2015

\section{References}

1. Vogt BA. Pain and emotion interactions in subregions of the cingulate gyrus. Nat Rev Neurosci. 2005;6:533-44.

2. Zhuo M. Cortical excitation and chronic pain. Trends Neurosci. 2008;31:199-207.

3. Dunckley P, Wise RG, Aziz Q, Painter D, Brooks J, Tracey I, et al. Cortical processing of visceral and somatic stimulation: differentiating pain intensity from unpleasantness. Neuroscience. 2005;133:533-42.

4. Peyron R, Laurent B, Garcia-Larrea L. Functional imaging of brain responses to pain. A review and meta-analysis (2000). Neurophysiol Clin. 2000;30:263-88.

5. Strigo IA, Duncan GH, Boivin M, Bushnell MC. Differentiation of visceral and cutaneous pain in the human brain. J Neurophys. 2003;89:3294-303.

6. Talbot JD, Marrett S, Evans AC, Meyer E, Bushnell MC, Duncan GH. Multiple representations of pain in human cerebral cortex. Science. 1991;251:1355-8.

7. Pillay PK, Hassenbusch SJ. Bilateral MRI-guided stereotactic cingulotomy for intractable pain. Stereotact Funct Neurosurg. 1992;59:33-8.

8. Wong ET, Gunes S, Gaughan E, Patt RB, Ginsberg LE, Hassenbusch SJ, et al. Palliation of intractable cancer pain by MRl-guided cingulotomy. Clin J Pain. 1997;13:260-3.

9. Yen CP, Kuan CY, Sheehan J, Kung SS, Wang CC, Liu CK, et al. Impact of bilateral anterior cingulotomy on neurocognitive function in patients with intractable pain. J Clin Neurosci. 2009;16:214-9.

10. Yen CP, Kung SS, Su YF, Lin WC, Howng SL, Kwan AL. Stereotactic bilateral anterior cingulotomy for intractable pain. J Clin Neurosci. 2005;12:886-90.

11. Zhuo M. Cortical plasticity as a new endpoint measurement for chronic pain. Mol Pain. 2011;7:54

12. Fuchs PN, Peng YB, Boyette-Davis JA, Uhelski ML. The anterior cingulate cortex and pain processing. Front Integr Neurosci. 2014;8:35.

13. Shyu BC, Vogt BA. Short-term synaptic plasticity in the nociceptive thalamic-anterior cingulate pathway. Mol Pain. 2009;5:51.

14. Johansen JP, Fields HL, Manning BH. The affective component of pain in rodents: direct evidence for a contribution of the anterior cingulate cortex. Proc Nat Acad Sci U S A. 2001;98:8077-82.

15. LaGraize SC, Labuda CJ, Rutledge MA, Jackson RL, Fuchs PN. Differential effect of anterior cingulate cortex lesion on mechanical hypersensitivity and escape/avoidance behavior in an animal model of neuropathic pain. Exp Neurol. 2004;188:139-48.

16. Koga K, Li X, Chen T, Steenland HW, Descalzi G, Zhuo M. In vivo whole-cell patch-clamp recording of sensory synaptic responses of cingulate pyramidal neurons to noxious mechanical stimuli in adult mice. Mol Pain. 2010;6:62.

17. Shyu BC, Chen WF, Shih HC. Electrically and mechanically evoked nociceptive neuronal responses in the rat anterior cingulate cortex. Acta Neurochir Suppl. 2008;101:23-5.

18. Sikes RW, Vogt BA. Nociceptive neurons in area 24 of rabbit cingulate cortex. J Neurophys. 1992;68:1720-32.

19. Wei F, Zhuo M. Potentiation of sensory responses in the anterior cingulate cortex following digit amputation in the anaesthetised rat. J Physiol. 2001;532:823-33.

20. Xu H, Wu LJ, Wang H, Zhang X, Vadakkan Kl, Kim SS, et al. Presynaptic and postsynaptic amplifications of neuropathic pain in the anterior cingulate cortex. J Neurosci. 2008;28:7445-53.

21. Li XY, Ko HG, Chen T, Descalzi G, Koga K, Wang H, et al. Alleviating neuropathic pain hypersensitivity by inhibiting PKMzeta in the anterior cingulate cortex. Science. 2010;330:1400-4.

22. Wang H, Xu H, Wu LJ, Kim SS, Chen T, Koga K, et al. Identification of an adenylyl cyclase inhibitor for treating neuropathic and inflammatory pain. Sci Transl Med. 2011;3:65ra63.

23. Wei F, Qiu CS, Kim SJ, Muglia L, Maas JW, Pineda W, et al. Genetic elimination of behavioral sensitization in mice lacking calmodulinstimulated adenylyl cyclases. Neuron. 2002;36:713-26.

24. Wu LJ, Toyoda H, Zhao MG, Lee YS, Tang J, Ko SW, et al. Upregulation of forebrain NMDA NR2B receptors contributes to behavioral sensitization after inflammation. J Neurosci. 2005;25:11107-16.

25. Calejesan AA, Kim SJ, Zhuo M. Descending facilitatory modulation of a behavioral nociceptive response by stimulation in the adult rat anterior cingulate cortex. Eur J Pain. 2000;4:83-96. 
26. Chen T, Wang W, Dong YL, Zhang MM, Wang J, Koga K, et al. Postsynaptic insertion of AMPA receptor onto cortical pyramidal neurons in the anterior cingulate cortex after peripheral nerve injury. Mol Brain. 2014;7:76.

27. Tye KM, Deisseroth K. Optogenetic investigation of neural circuits underlying brain disease in animal models. Nat Rev Neurosci. 2012;13:251-66.

28. Yizhar O, Fenno LE, Davidson TJ, Mogri M, Deisseroth K. Optogenetics in neural systems. Neuron. 2011;71:9-34.

29. Van den Oever MC, Rotaru DC, Heinsbroek JA, Gouwenberg Y, Deisseroth K, Stuber GD, et al. Ventromedial prefrontal cortex pyramidal cells have a temporal dynamic role in recall and extinction of cocaine-associated memory. J Neurosci. 2013;33:18225-33.

30. Wolff SB, Grundemann J, Tovote P, Krabbe S, Jacobson GA, Muller C, et al. Amygdala interneuron subtypes control fear learning through disinhibition. Nature. 2014;509:453-8.

31. Iyer SM, Montgomery KL, Towne C, Lee SY, Ramakrishnan C, Deisseroth K et al. Virally mediated optogenetic excitation and inhibition of pain in freely moving nontransgenic mice. Nat Biotechnol. 2014;32:274-8

32. Hickey L, Li Y, Fyson SJ, Watson TC, Perrins R, Hewinson J, et al. Optoactivation of locus ceruleus neurons evokes bidirectional changes in thermal nociception in rats. J Neurosci. 2014;34:4148-60.

33. Lee M, Manders TR, Eberle SE, Su C, D'Amour J, Yang R, et al. Activation of corticostriatal circuitry relieves chronic neuropathic pain. J Neurosci. 2015;35:5247-59

34. Wang GQ, Cen C, Li C, Cao S, Wang N, Zhou Z, et al. Deactivation of excitatory neurons in the prelimbic cortex via Cdk5 promotes pain sensation and anxiety. Nat Commun. 2015;6:7660.

35. Zhang Z, Gadotti VM, Chen L, Souza IA, Stemkowski PL, Zamponi GW. Role of Prelimbic GABAergic Circuits in Sensory and Emotional Aspects of Neuropathic Pain. Cell Rep. 2015;12:752-9.

36. Barthas F, Sellmeijer J, Hugel S, Waltisperger E, Barrot M, Yalcin I. The anterior cingulate cortex is a critical hub for pain-induced depression. Biol Psychiatry. 2015;77:236-45

37. Gu L, Uhelski ML, Anand S, Romero-Ortega M, Kim YT, Fuchs PN, et al. Pain inhibition by optogenetic activation of specific anterior cingulate cortical neurons. Plos One. 2015:10, e0117746

38. Chen T, Koga K, Descalzi G, Qiu S, Wang J, Zhang LS, et al. Postsynaptic potentiation of corticospinal projecting neurons in the anterior cingulate cortex after nerve injury. Mol Pain. 2014;10:33.

39. Delevich K, Tucciarone J, Huang ZJ, Li B. The Mediodorsal Thalamus Drives Feedforward Inhibition in the Anterior Cingulate Cortex via Parvalbumin Interneurons. J Neurosci. 2015;35:5743-53.

\section{Submit your next manuscript to BioMed Central and we will help you at every step:}

- We accept pre-submission inquiries

- Our selector tool helps you to find the most relevant journal

- We provide round the clock customer support

- Convenient online submission

- Thorough peer review

- Inclusion in PubMed and all major indexing services

- Maximum visibility for your research 